\title{
Symmetry breaking in $\mathrm{O}_{4}^{+}$: an application of the Brueckner coupled-cluster method
}

\author{
Leslie A. Barnes* \\ Eloret Institute \\ Palo Alto, CA 94303, USA \\ and \\ Roland Lindh \\ Dept. of Theoretical Chemistry \\ University of Lund \\ Chemical Centre \\ P. O. Box 124 \\ S-221 00 Lund, Sweden
}

\section{Abstract}

A recent calculation of the antisymmetric stretch frequency for the rectangular structure of quartet $\mathrm{O}_{4}^{+}$using the QCISD(T) method gave a value of $3710 \mathrm{~cm}^{-1}$. This anomalous frequency is shown to be a consequence of symmetry breaking effects, which occur even though the QCISD(T) solution derived from a delocalized SCF reference function lies energetically well below the two localized (symmetry-broken) solutions at the equilibrium geometry. The symmetry breaking is almost eliminated at the CCSD level of theory, but the small remaining symmetry breaking effects are magnified at the $\operatorname{CCSD}(\mathrm{T})$ level of theory so that the antisymmetric stretch frequency is still significantly in error. The use Brueckner coupled cluster method, however, leads to a symmetrical solution which is free of symmetry breaking effects, with an antisymmetric stretch frequency of $1322 \mathrm{~cm}^{-1}$, in good agreement with our earlier calculations using the CASSCF/CASSI method.

* Mailing address: NASA Ames Research Center, Moffett Field, California 94035-1000

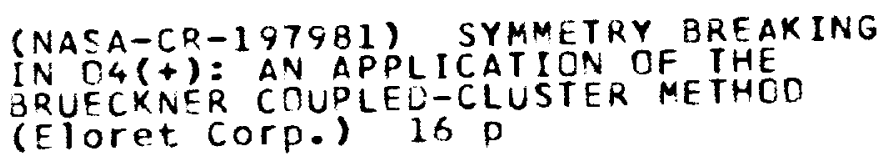




\section{Introduction}

Recently, Peel [1] reported $a b$ initio calculations on some high symmetry isomers of quartet $\mathrm{O}_{4}^{+}$using the singles and doubles quadratic configuration interaction method with a perturbational estimate of connected triple excitations (QCISD(T)) [2]. For the rectangular structure, he obtained an antisymmetric stretch frequency of $3710 \mathrm{~cm}^{-1}$, which he noted was "unphysical", but nevertheless was used to give a large zero-point correction to the energy of this isomer.

Previously [3], we had carried out a detailed study of several structures of quartet $\mathrm{O}_{4}^{+}$, computing amongst other things vibrational frequencies, relative energies and isotopic shifts. The methods used included complete active space self consistent field (CASSCF) [4], restricted active space self consistent field (RASSCF) [5], a second order perturbation method based on a CASSCF reference function (CASPT2) [6] and a non-orthogonal CI method based on non-orthogonal CASSCF or RASSCF solutions (the complete/restricted active space state interaction (CASSI/RASSI) method [7]), using large generally contracted atomic natural orbital basis sets. These methods gave good results and prompted a new analysis [8] of the experimental vibrational spectrum of $\mathrm{O}_{4}^{+}$, which supported our assignment. Of particular importance for the current work is our detailed analysis of symmetry breaking effects in the calculation of the antisymmetric stretch frequency for quartet $\mathrm{O}_{4}^{+}$. We showed that spurious frequencies for quartet $\mathrm{O}_{4}^{+}$were due to symmetry breaking effects due to the competition between localized and delocalized structures - we refer the reader to our original work [3], where we also give many references to earlier work on symmetry breaking. In our case, the symmetry breaking effects were resolved through the use of the CASSI method, allowing the two non-orthogonal "localized" CASSCF wavefunctions to interact and give the correct qualitative form for the potential energy surface.

On the basis of the form of the potential curve for the antisymmetric stretch around the equilibrium point for the rectangular structure, Peel [1] concluded that no symmetry breaking effects were evident at the QCISD $(T)$ level of theory. In the first part of this work we demonstrate that this is incorrect: symmetry breaking effects are entirely responsible for the anomalous antisymmetric stretch frequency of $3710 \mathrm{~cm}^{-1}$. For consistency, we use the same methods as in Ref. [1] - QCISD(T) calculations based on an unrestricted Hartree Fock (UHF) reference function and a 6-31G* basis set. The geometry $\left(R_{0 O}=1.186 \AA, R_{C M}=2.378 \AA\right.$ ) was also taken from Ref. [1]. Roo is the intra-fragment $\mathrm{O}-\mathrm{O}$ bond distance, $\mathrm{R}_{\mathrm{CM}}$ is the inter-fragment bond distance (the distance between the center of masses of the two fragments). The rectangular structure is illustrated in Fig. 1(a), which also shows $R_{\mathrm{OO}}$ and $\mathrm{R}_{\mathrm{CM}}$.

Following this, we give the results of calculations at the $\operatorname{CCSD}(\mathrm{T})$ level of theory (singles and doubles coupled-cluster plus a perturbational estimate of the effects of connected triples excitations [9]). The geometry is reoptimized and from the computed potential curves and frequencies it is evident that symmetry breaking effects also occur at the $\operatorname{CCSD}(\mathrm{T})$ level of theory. Brueckner coupled cluster theory [10]-[15] has previously been used to treat symmetry breaking effects at the coupled cluster level of theory [16]. We 
have applied this approach to the rectangular structure of quartet $\mathrm{O}_{4}^{+}$, using Brueckner coupled cluster calculations which include a perturbational estimate of the effects of connected triples excitations $(B D(T))$ [14], with semi-canonical orbitals [17]. Since we are interested in the qualitative nature of the results, rather than strictly quantitative results, we continue to use the $6-31 \mathrm{G}^{*}$ basis set and UHF reference function, rather than the larger generally contracted basis sets of our earlier study [3]. We note that in the $\operatorname{CCSD}(\mathrm{T})$ and $\mathrm{BD}(\mathrm{T})$ calculations, all electrons are correlated, whereas in the QCISD and QCISD(T) calculations, the $1 s$ core electrons were not correlated.

The calculations were performed with the ACES II ${ }^{1}$ suite of programs using IBM RISC SYSTEM/6000 computers at NASA Ames Research Center.

\section{Results and Discussion}

As discussed in detail in our previous work [3], the symmetry breaking in $\mathrm{O}_{4}^{+}$is manifested by the existence of a delocalized solution, which exhibits $D_{2 h}$ symmetry, and two localized solutions exhibiting $\mathrm{C}_{2 v}$ symmetry which are mirror images of each other, having equal energies at the symmetric $\left(D_{2 h}\right)$ point. The structures are illustrated in Fig. 1 , with Fig. 1(a) showing the symmetric geometry, and Figs. 1(b) and (c) showing the two symmetry broken localized solutions. In each case, the shorter $\mathrm{O}-\mathrm{O}$ bond distance corresponds closely to the bond distance of $\mathrm{O}_{2}^{+}$, whereas the longer bond distance corresponds closely to the bond distance of $\mathrm{O}_{2}$ [3]. Thus the positive charge is localized on the bottom $\mathrm{O}_{2}$ unit in Fig. 1(b), and on the top $\mathrm{O}_{2}$ unit in Fig. 1(c). The symmetry breaking vibrational mode is the intra-fragment antisymmetric stretch $\omega_{5}$, and this is illustrated in Figs. 1(b) and (c) by the arrows.

In Table I, we give the total energies of the different solutions at the UHF, QCISD and QCISD(T) levels of theory. Comparing the total energy at the QCISD(T) level with that given in Ref. [1], we see that the results of Ref. [1] are based on the delocalized reference function. It is interesting to compare the energy differences of the delocalized and localized solutions at the various levels of theory. At the UHF and QCISD levels of theory, the localized solution is below the delocalized solution by about $3 \mathrm{kcal} / \mathrm{mol}$. However, at the QCISD(T) level of theory, the localized solution is more than $7 \mathrm{kcal} / \mathrm{mol}$ higher in energy than the delocalized solution. Thus it seems that the $\mathrm{D}_{2 h}$ structure is favoured at the highest level of theory.

To understand the origin of the spurious frequency at the QCISD(T) level of theory, we have computed the energy as a function of the antisymmetric stretch coordinate (see

\footnotetext{
${ }^{1}$ ACES II is a computational chemistry package especially designed for coupled cluster and many body perturbation calculations. The SCF, transformation, correlation energy and gradient codes were written by J. F. Stanton, J. Gauss, J. D. Watts, W. J. Lauderdale and R. J. Bartlett. The two-electron integrals are taken from the vectorized MOLECULE code of J. Almlöf and P. R. Taylor. ACES II includes a modified version of the ABACUS integral derivatives program, written by T. Helgaker, H. J. Jensen, $P$. Jørgensen, J. Olsen, and P. R. Taylor, and the geometry optimization and vibrational analysis package written by J. F. Stanton and D. E. Bernholdt.
} 
also Refs. [1] and [3]). The results are presented in Figs. 2-4, where we have used the same scales in order to facilitate comparison of the different curvatures resulting from the different methods. The delocalized solution is given by $E_{\text {del }}$ and the two localized solutions are given by $E_{a}$ and $E_{b}$ in each case. Also given in Figs. 3 and 4 are curves at the $\operatorname{CCSD}, \operatorname{CCSD}(\mathrm{T}), \mathrm{BD}$ and $\mathrm{BD}(\mathrm{T})$ level of theory, which we discuss later. Using the notation of Figs. $1(b)$ and $(c), \Delta R$ is defined as $R_{1}-R_{2}$.

The behaviour of the energy at the correlated level of theory is driven by the behaviour of the UHF energies, given in Fig. 2. The delocalized solution lies above the localized solutions, and as we follow the antisymmetric coordinate, the delocalized solution rapidly approaches the localized solutions, until at $\Delta R \approx 0.0185 \AA$ the localized and delocalized solutions merge. Thus the QCISD and QCISD $(T)$ energies are constrained by this fact - the delocalized energy and one of the localized energies (depending on whether the distortion is positive or negative) must be equal at $\Delta R \approx 0.0185 \AA$. In Fig. 3, we see that at the QCISD level of theory the energies are significantly better in the sense that the localized solution energies are much flatter (tending towards delocalized solutions). In this case the flatness of the localized solutions means that the delocalized solution is driven down in energy in order to meet the constraint of equal energy at $\Delta R \approx 0.0185 \AA$, leading to an imaginary frequency at the QCISD level of theory. At the QCISD(T) level of theory (Fig. 4) the delocalized solution is well below the localized solution, and so is driven rapidly upward to meet the equal energy constraint, resulting in the very large $3710 \mathrm{~cm}^{-1}$ frequency. Thus the QCISD method does not entirely overcome the inherent problems with the UHF reference function, and the triples perturbation correction is unable to overcome the residual problems with the QCISD method. We note that we found similar problems with the CASPT2 method, which was not able to overcome the problems of a localized CASSCF reference function [3].

In Tables II and III we present the results from the $\operatorname{CCSD}(\mathrm{T})$ calculations. From the energy separations, we see that while the UHF separation is very similar to that given in Table I (which has a slightly different geometry), the CCSD and CCSD(T) separations are very different to those at the QCISD and QCISD $(T)$ levels of theory. The delocalized and localized solutions are very close in energy at the CCSD level of theory, and unlike the QCISD results the localized solution is above the delocalized solution. As for the QCISD(T) results, the perturbational triples correction increases the separation between the delocalized and localized solutions at the $\operatorname{CCSD}(\mathrm{T})$ theory when compared with CCSD, although the effect is much smaller than the QCISD and QCISD(T) difference. We note that one difference between the QCISD and CCSD calculations was that the $1 s$ core electrons were excluded from the calculations at the QCISD and QCISD(T) levels of theory, whereas they were included in the calculations at the CCSD and CCSD(T) levels of theory. To check whether this difference has any effect on the symmetry breaking at the QCISD/QCISD(T) level of theory, we also computed the separation between the delocalized and localized solutions at the symmetric point including the $1 s$ electrons using these methods. The separations are barely different from the original results, so we conclude that removing the core $1 s$ electrons from the QCISD calculations is not the cause of the large difference between the $\operatorname{CCSD} / \operatorname{CCSD}(\mathrm{T})$ and $\mathrm{QCISD} / \mathrm{QCISD}(\mathrm{T})$ results.

In Figs. 3 and 4 we present the CCSD and $\operatorname{CCSD}(T)$ potential curves for the antisymmetric 
stretch, which may be compared with the QCISD and QCISD(T) curves on the same figures. The behaviour of the UHF reference function energies around the $\operatorname{CCSD}(\mathrm{T})$ equilibrium geometry for the antisymmetric stretch is very similar to that given in Fig. 2 around the QCISD(T) equilibrium geometry, so we may discuss the CCSD and CCSD(T) curves in the same light as the QCISD and QCISD(T) curves. Thus the CCSD and $\operatorname{CCSD}(\mathrm{T})$ energies have similar constraints to the QCISD and QCISD(T) energies irrespective of the separation at $\Delta R=0$ (the symmetric geometry), at $\Delta R \approx 0.0185 \AA$ the delocalized and one of the localized energies must be equal. Inspection of the curves shows that this is so. However, the most striking difference between the CCSD and QCISD curves comes from the fact that the curves at the CCSD level are much closer together, so that this constraint has only a small effect on the antisymmetric stretch frequency at the CCSD level of theory. In fact, the antisymmetric stretch frequency at the CCSD level of theory is a very reasonable $1220 \mathrm{~cm}^{-1}$ (at the CCSD equilibrium geometry), which is to be compared with a value of around $1500 i \mathrm{~cm}^{-1}$ at the QCISD level of theory. Overall, we see that the CCSD approach has almost eliminated the symmetry breaking effects.

As discussed above, the addition of the perturbative triples correction increases the separation between the delocalized and localized solutions at the symmetric point, and this is evident in Fig. 4. Thus the delocalized curve at the CCSD(T) level is more affected by symmetry breaking than the CCSD curve, although this effect is much smaller than that found with the QCISD(T) method. Thus the antisymmetric stretch frequency at the $\operatorname{CCSD}(\mathrm{T})$ level is $1922 \mathrm{~cm}^{-1}$, compared with $3710 \mathrm{~cm}^{-1}$ at the QCISD(T) level of theory. The origin of this difference is quite evident from the potential curves - it is the large difference in separations at the symmetric point. The other remarkable feature of the $\operatorname{CCSD}(T)$ potential curves is the near coincidence of the two localized curves, which is again quite different to the $\mathrm{QCISD}(\mathrm{T})$ results. Thus the $\operatorname{CCSD}(\mathrm{T})$ approach is quite close to removing the symmetry breaking effects, but is still not able to overcome the small deficiencies evident at the CCSD level of theory.

The geometry at the $\operatorname{CCSD}(\mathrm{T})$ level (Table III) is very similar to that found at the $\mathrm{QCISD}(\mathrm{T})$ level of theory [1], and for the most part the frequencies are quite similar to those given in Ref. [1]. The exceptions are the antisymmetric stretch $\omega_{5}$ (discussed above) which changes from 3710 to $1922 \mathrm{~cm}^{-1}$, and the (inter-fragment) antisymmetric stretch $\omega_{6}$ which is reduced from 595 to $97 \mathrm{~cm}^{-1}$. The $\operatorname{CCSD}(\mathrm{T})$ value for $\omega_{6}$ is in accord with our earlier results [3] and the results for the trans-planar structure $[1,3]$. Thus it seems that the QCISD(T) value for $\omega_{6}$ is significantly too high also. Considering Fig. 1, the mode $\omega_{6}$ may be envisioned in an analogous way to $\omega_{5}$, except that the distortion occurs along the $\mathrm{R}_{\mathrm{CM}}$ direction instead of the Roo direction. Thus it is possible, though less likely (due to the large inter-fragment bond distance $R_{\mathrm{CM}}$ ), for symmetry breaking effects to occur for $\omega_{6}$ also. This would involve localization on the left and right sides of $\mathrm{O}_{4}^{+}$rather than the top and bottom, which occurs for $\omega_{5}$. However, we have not investigated this in any detail here.

The results at the $\mathrm{BD}(\mathrm{T})$ level of theory are given in Table IV. The geometry optimized at the $\mathrm{BD}(\mathrm{T})$ level of theory is the same as that of the $\operatorname{CCSD}(\mathrm{T})$ level of theory (which was constrained to have $\mathrm{D}_{2 h}$ symmetry, whereas the $\mathrm{BD}(\mathrm{T})$ calculation was not), and the $\mathrm{BD}(\mathrm{T})$ energy is also very similar to the $\operatorname{CCSD}(\mathrm{T})$ energy. To investigate whether 
symmetry breaking effects are still present at the $\mathrm{BD}(\mathrm{T})$ level of theory, we have again plotted the energy as a function of the antisymmetric distortion, and the results are given in Figs. 3-5.

As we saw previously, the behaviour of the correlated methods was constrained by the behaviour of the reference function. In Fig. 5 we give the Brueckner reference determinant energy and the UHF energy from which the Brueckner calculation was initiated. Before discussing these results, we emphasize that the comparison between the Brueckner reference determinant energy and the UHF energy is not rigourous since the Brueckner reference determinant is a product of the correlated calculation. Nevertheless, it is enlightening.

The UHF solutions of Fig. 5 are (qualitatively speaking) a subset of those given in Fig. 2 . The curve is discontinuous because we varied $\Delta R$ with a larger stepsize than for Fig. 2, and the SCF converged to solutions on different potential curves at different points, rather than the solution on the same potential curve as in Fig. 2. The character of the UHF orbitals is of course very different for the different potential energy surfaces, varying from delocalized to localized on the top of the molecule or localized on the bottom of the molecule, and this variation is reflected in the energies. In contrast to this, the Brueckner reference energy is very smooth despite the large changes in the UHF orbitals from which it began, indicating that the Brueckner approach is not affected by the starting orbitals. At the symmetric point we have also verified that the Brueckner method is independent of the starting orbitals - whether localized or delocalized UHF orbitals are used, the Brueckner approach leads to the same symmetric (delocalized) solution. Thus there is only one solution at the Brueckner level of theory. This behaviour is in accord with previous studies [16] using the Brueckner approach for other systems which exhibit symmetry breaking.

At the $\mathrm{BD}$ and $\mathrm{BD}(\mathrm{T})$ levels of theory (Figs. 3 and 4 ) we see that the antisymmetric stretch is very smooth and gives a positive frequency, which is $1322 \mathrm{~cm}^{-1}$ at the $\mathrm{BD}(\mathrm{T})$ level of theory (Table IV). It is interesting to compare the different curvatures for the different methods in Figs. 3 and 4. It is evident that the CCSD and CCSD(T) curvatures are much closer to the $\mathrm{BD}$ and $\mathrm{BD}(\mathrm{T})$ curves than are those from QCISD and QCISD(T).

We note from our previous study [3] that there is a significant basis set effect for the antisymmetric stretch frequency. At the CASSI level using a TZ2P basis set, the antisymmetric stretch was $1271 \mathrm{~cm}^{-1}$ whereas an ANO[5s4p2d] basis set gave a value of $1259 \mathrm{~cm}^{-1}$ and an ANO[6s5p3d2f] basis gave a value of $1296 \mathrm{~cm}^{-1}$. Considering the fact that the $\mathrm{BD}(\mathrm{T})$ approach should give a larger proportion of the dynamical correlation energy than our earlier frequency calculations at the CASSCF/CASSI level of theory, the agreement between the $\mathrm{BD}(\mathrm{T})$ frequency and our earlier values is very good. Thus the $\mathrm{BD}(\mathrm{T})$ results are very encouraging and in a large one particle basis this method should give very accurate results. In our earlier work [3] we showed that the dipole derivative at the CASSI level of theory was unphysically high. It would be of some interest to compute this quantity at the $\mathrm{BD}(\mathrm{T})$ level (in a large one particle basis) to determine whether a more reasonable dipole derivative would be obtained.

In Table IV we also give the symmetric stretch frequencies at the $\mathrm{BD}(\mathrm{T})$ level of theory. 
Given the agreement between the geometries at the $\operatorname{CCSD}(\mathrm{T})$ and $\mathrm{BD}(\mathrm{T})$ levels of theory, it is not surprising that the symmetric stretch frequencies are very similar for the two methods (and also in good agreement with the CASSCF results [3]). These results also support our earlier isotopic substitution analysis [3], where we used the CASSCF frequency for the symmetric stretch and the CASSI frequency for the antisymmetric stretch.

To conclude, the antisymmetric stretch of quartet $\mathrm{O}_{4}^{+}$is significantly affected by symmetry breaking. As we discussed previously [3], it is necessary to properly account for this before a reliable frequency can be obtained. In the current work we have shown in detail how the previous [1] antisymmetric stretch frequency at the QCISD $(\mathrm{T})$ level of theory is affected by symmetry breaking so that any analysis of the relative energies of the rectangular and trans-planar structures which includes zero-point corrections based on this frequency must be significantly in error. The CCSD approach gives significantly better results than QCISD, almost eliminating the symmetry breaking effects. However, the small remaining symmetry breaking effects are magnified at the $\operatorname{CCSD}(\mathrm{T})$ level of theory, so that the antisymmetric stretch is still affected significantly at the $\operatorname{CCSD}(\mathrm{T})$ level. The Brueckner coupled-cluster method $(\mathrm{BD}(\mathrm{T}))$, however, eliminates the symmetry breaking effects entirely, giving a single symmetric solution with an antisymmetric stretch frequency in good agreement with our earlier result at the CASSCF/CASSI level of theory [3]. This must make the $\mathrm{BD}(\mathrm{T})$ approach the method of choice for very accurate calculations when symmetry breaking is a potential problem and more than just a few electrons must be correlated.

\section{Acknowledgements}

L.A.B. was supported by NASA grant number NCC-2-741, and R.L. was supported by a grant from the Swedish Natural Research Council (NFR) and by IBM Sweden under a joint study contract. The receipt of a preprint of Ref. [1] is acknowledged. 


\section{References}

[1] J. B. Peel, Chem. Phys. Lett. 218, 367 (1994).

[2] J. A. Pople, M. Head-Gordon, and K. Raghavachari, J. Chem. Phys. 87, 5968 (1987).

[3] R. Lindh and L. A. Barnes, J. Chem. Phys. 100, 224 (1994).

[4] P.- $\AA$. Malmqvist, A. Rendell, and B. O. Roos, J. Chem. Phys. 94, 5477 (1990), and references therein.

[5] J. Olsen, B. O. Roos, P. Jørgensen, and H. J. Aa. Jensen J. Chem. Phys. 89, 2185 (1985).

[6] K. Andersson, P.-Å. Malmqvist, B. O. Roos, A. J. Sadlej, and K. Wolinski, J. Chem. Phys. 94, 5483 (1990); K. Andersson, P.- $\AA$. Malmqvist, and B. O. Roos, ibid 96, 1218 (1992).

[7] P.- $\AA$. Malmqvist, Int. J. Quant. Chem. 30, 479 (1986); P.- $\AA$. Malmqvist and B. O. Roos, Chem. Phys. Lett. 155, 189 (1989).

[8] M. E. Jacox and W. E. Thompson, J. Chem. Phys. 100, 750 (1994).

[9] K. Raghavachari, G. W. Trucks, J. A. Pople, and M. Head-Gordon Chem. Phys. Lett. 157, 479 (1989).

[10] K. A. Brueckner, Phys. Rev. 96, 508 (1954).

[11] R. K. Nesbet, Phys. Rev. 109, 1632 (1958).

[12] R. A. Chiles and C. E. Dykstra, J. Chem. Phys., 74, 4544 (1981).

[13] L. Z. Stolarczyk and H. J. Monkhorst, Int. J. Quantum Chem. Symp. 18, 267 (1984).

[14] N. C. Handy, J. A. Pople, M. Head-Gordon, K. Raghavachari, and G. W. Trucks Chem. Phys. Lett. 164, 185 (1989).

[15] K. Raghavachari, J. A. Pople, E. S. Replogle, M. Head-Gordon, and N. C. Handy, Chem. Phys. Lett. 167, 115 (1990).

[16] J. F. Stanton, J. Gauss, and R. J. Bartlett, J. Chem. Phys. 97, 5554 (1992).

[17] J. D. Watts, J. Gauss, and R. J. Bartlett, J. Chem. Phys. 98, 8718 (1993). 
Table I: Total energies (in Hartree) and energy differences (in $\mathrm{kcal} / \mathrm{mol}$ ) of quartet $\mathrm{O}_{4}^{+}$at the rectangular $\left(\mathrm{D}_{2 h}\right)$ geometry, for the delocalized and localized solutions. The geometry is taken from the QCISD(T) calculations of Ref.[1] $\left(\mathrm{R}_{\mathrm{OO}}=1.186 \AA \mathrm{R}_{\mathrm{CM}}=2.378 \AA\right.$ )

\begin{tabular}{|l|c|c|r|}
\hline \hline Method & $\mathrm{E}_{\text {del }}$ & $\mathrm{E}_{\text {loc }}$ & $\Delta \mathrm{E}$ \\
\hline \hline UHF & -298.7398444 & -298.7439668 & 2.59 \\
QCISD & -299.4774306 & -299.4830304 & 3.51 \\
QCISD(T) & -299.5050169 & -299.4933392 & -7.33 \\
\hline
\end{tabular}

Table II: Total energies (in Hartree) and energy differences (in $\mathrm{kcal} / \mathrm{mol}$ ) of quartet $\mathrm{O}_{4}^{+}$at the rectangular $\left(D_{2 h}\right)$ geometry, for the delocalized and localized solutions. The geometry is from the $\operatorname{CCSD}(\mathrm{T})$ approach, given in Table III

\begin{tabular}{|l|c|c|r|}
\hline \hline Method & $\mathrm{E}_{\text {del }}$ & $\mathrm{E}_{\text {loc }}$ & $\Delta \mathrm{E}$ \\
\hline \hline UHF & -298.7406630 & -298.7447092 & 2.54 \\
CCSD & -299.4838322 & -299.4832236 & -0.38 \\
CCSD $(\mathrm{T})$ & -299.5127862 & -299.5107674 & -1.27 \\
\hline \hline
\end{tabular}

